\title{
Tea Leaves Moisture Prediction Using One-Port Monopole Sensor
}

\author{
J. A. Ling ${ }^{1}$, Y. L. Then ${ }^{2 *}$, K. Y. You ${ }^{3}$, J. Ahmed ${ }^{4}$, and D. N. A. Zaidel ${ }^{5}$ \\ ${ }^{1,2,4}$ Department of Electrical and Electronics Engineering, Faculty of Engineering, Computing and Science, \\ Swinburne University of Technology, 93350 Kuching, Sarawak. \\ ${ }^{3}$ Department of Communication Engineering, Faculty of Electrical Engineering, Universiti Teknologi Malaysia, \\ UTM Skudai 81310, Malaysia. \\ ${ }^{5}$ Department of Electrical and Electronics Engineering, Faculty of Engineering, Universiti Malaysia Sarawak, \\ 94300, Kota Samarahan, Sarawak. \\ *Corresponding author: ythen@swinburne.edu.my
}

\begin{abstract}
This paper presents the application of using a coaxial monopole sensor for determining and formulating the relationship between the reflection coefficient and the moisture content of tea leaves. A coaxial monopole sensor with operating frequency ranges from $1.35 \mathrm{GHz}$ to $1.95 \mathrm{GHz}$ was used. The data collections were conducted by using the Vector Network Analyzer. Visual comparison of the results between the actual and predicted gravimetric moisture content are presented. A predictive model is being generated and tested. It is indicated that the monopole sensor has an excellent accuracy of $98.76 \%$ and $\mathbf{9 9 . 5 9 \%}$ in predicting the gravimetric and volumetric moisture content in the sample, respectively. This research has shown that monopole sensor is applicable for the determination of moisture content in tea leaves as an optional sensing technique.
\end{abstract}

Keywords-Moisture; Monopole; Sensor; Microwave; Reflection coefficient.

\section{INTRODUCTION}

In the recent years of technology advancement, the use of sensor technology has been widely accepted by many as a method in industry as well as research facility for determine the contents in samples, especially in this case would be m.c. of the samples. The determination of m.c.in the samples is an important task as it is one of the predominant factors that would affect the quality of the product. Moisture Content detection can be seen as an important assessment in the determination of various food and agricultural product. Moreover, in the past technology advancement, various type of method to determine m.c. has been developed and used. In this project, it is to determine the relationship of the reflection coefficient and the moisture content, m.c. of the tea leaves sample and also to determine the quality of local tea leaves product by using a monopole sensor probe. The operational frequency used in the project would be $1.35 \mathrm{GHz}$ to $1.95 \mathrm{GHz}$. Also, an indirect analysis which involve in using the gravimetric and volumetric method would be applied to measure the amount of m.c. in the sample.

Monopole sensor is chosen for its high precision advantage as it has high penetration rate that would give a high dynamic range of the reflection coefficient[1] and also smaller in size. In a study done, the monopole antenna was used in the determination of m.c. of Dioscorea hispida tubers. It consists of using a VNA in the measuring of the reflection coefficient while the monopole sensor was inserted into the D.hispida tuber and the tuber is applied with different percentage of moisture. The optimal operation frequency for observing the relationship between the reflection coefficient and the D.hispida tuber was found to be $0.8 \mathrm{GHz}$. Furthermore, it is also stated in this particular research paper that by using oven drying method can assist in the determination of the true m.c. of the samples[1]. Moreover, in one of the research on using microwave sensing technique, a sub-domain method of moment formulation was proposed to model the problem of an open-ended coaxial probe radiating into a metal with surface-braking crack of arbitrary depth profile. Moreover, this unique technique that will be used in the crack detection. The proposed modelling technique will see an arbitrary-shape crack as a series of rectangular waveguides and utilizes the generalized scattering matrix method to obtain the reflection coefficient of the incident field [2].

In another study done, it describes the accurate determination of the Moisture Contents of Organic soils using the oven drying method. The factors that affect the accurate determination of the moisture content values in organic soils are depends on the temperature that was set on the oven when drying the sample. Moreover, it is also mentioned that a routine moisture test should be conducted to identify the changes in the sample. Furthermore, it is also stated that the true m.c. calculations equal to the potential reduction in the mass of the sample with the evaporation of the waters[3].Based on another research done, it describes the use of using the microwave free-space technique for the measurement of moisture in the green tea sample. The finding from this paper has mentioned that by using this method has provided m.c. of the tea leaves from approximately $6 \%$ to $32 \%$ on a dry basis without any prior information on the thickness and the density of the given tea leaves sample. 